\title{
Development of a novel methodology for the analysis of ergosterol in mushrooms
}

\section{Running title: Novel methodology for ergosterol analysis}

João C.M. Barreira · M. Beatriz P.P. Oliveira · Isabel C.F.R. Ferreira

João C.M. Barreira · Isabel C.F.R. Ferreira $(\bowtie)$

CIMO/Escola Superior Agrária, Instituto Politécnico de Bragança, Apartado 1172, 5301-855 Bragança, Portugal

e-mail: iferreira@ipb.pt; telephone+351-273-303219; fax +351-273-325405

João C.M. Barreira · M. Beatriz P.P. Oliveira

REQUIMTE, Departamento de Ciências Químicas, Faculdade de Farmácia da Universidade do Porto, Rua Jorge Viterbo Ferreira, 228, 4050-313 Porto, Portugal. 


\begin{abstract}
Sterols are important molecules in the unsaponifiable fraction of several matrices. Ergosterol, which is an important vitamin $\mathrm{D}_{2}$ precursor, is clearly the main sterol in mushrooms. Herein, an analytical method for ergosterol determination in cultivated and wild mushrooms was developed using high performance liquid chromatography coupled to ultraviolet detection. The chromatographic separation was achieved in a Inertsil 100A ODS-3 reverse phase column using an isocratic elution with acetonitrile:methanol $(70: 30, v: v)$ at a flow rate of $1 \mathrm{~mL} / \mathrm{min}$. Different extraction methodologies were tested, using $n$-hexane, methanol:dichloromethane $(75: 25, v: v)$ or chloroform:methanol $(20: 10$, $v: v)$. The method was optimized using Fistulina hepatica and proved to be reproductive and accurate. Ergosterol was the most abundant sterol by a greater extent in all mushrooms. In general, the cultivated species showed higher contents, mainly Agaricus bisporus and Lentinus edodes. Among wild species, Boletus edulis was the mushroom with the highest content in ergosterol. Results were expressed in fat content, dry weight and fresh weight bases. The assessment of ergosterol amounts might be very useful due to the bioactive potential that has been attributed to this molecule and its derivatives.
\end{abstract}

Keywords: Ergosterol, analysis optimization, HPLC-UV, mushrooms. 


\section{Introduction}

Sterols can be found in nature as free or conjugate forms. In the latter, the $3 \beta$-hydroxyl group is esterified with a fatty acid or a hydroxycinnamic acid, or glycosylated with a hexose (usually glucose) or a 6-fatty acyl hexose (Moreau et al. 2002).

Phyto- and mycosterols are biosynthesized in similar pathways, but the sequence of postsqualene reactions and the stereochemistry of the major products diverge. Higher fungi produce 5,7,22-ergostatrien-3 $\beta$-ol (ergosterol) as the primary sterol (Mattila et al. 2002; Senatore 1992; Teichmann et al. 2007; Villares et al. 2012) with the two double bonds in the sterol ring structure instead of the single one specific from plants (Parks and Weete 1991). Nevertheless, ergosterol is also found in relatively high amounts in plants like corn, cotton seed, peanut and linseed oils (Kritchevsky and Shirley 2005; Lagarda et al. 2006). This sterol is becoming an important research topic because its content is usually associated with structural and growing fungal characteristics, such as maturation, hyphal formation and sporulation (Villares et al. 2012), being also dependent on the fungal species (Phillips et al. 2011). From the bioactivity point of view, ergosterol has great importance since it can be converted into vitamin $D_{2}$ after photolysis and thermal rearrangement (Villares et al. 2012), and was reported as having health-promoting properties such as antioxidant (Shao et al. 2010), anti-inflammatory (Kuo et al. 2011) and antihyperlipidemic (Hu et al. 2006) activities, being also involved in the activated expression of specific defense genes (Lochman and Mikes 2005).

Typically, the analysis of individual sterols includes the extraction of lipids, saponification (which might include a previous acid hydrolysis), extraction of unsaponifiable matter and separation/partial purification of sterols. However, other unsaponifiable compounds like squalene and other linear hydrocarbons might interfere 
in sterols analysis demanding a separation step usually achieved by chromatographic techniques, e.g., thin layer chromatography or solid phase extraction, formation of sterol derivatives and their analysis by capillary gas chromatography (Lagarda et al. 2006) or gas-liquid chromatography (GLC), which requires a derivatization step with trimethylsilane (Giacometti 2001). A suitable resolution might also be obtained with high performance liquid chromatography (HPLC), which is faster than GLC analysis and operates under milder column temperatures and non-destructive detection conditions (Bada et al. 2004; Manzi et al. 1996).

Chantarellus cibarius, Craterellus tubaeformis, Boletus edulis (Mattila et al. 2002; Teichmann et al. 2007), Lactarius trivialis (Mattila et al. 2002), Cordyceps sinensis (Yuan et al. 2007) and other Basidiomycetes (Senatore 1992) are amongst the wild mushroom species previously studied for their contents in ergosterol. Regarding cultivated species, similar studies were conducted using Pleurotus ostreatus, Agaricus bisporus/brown, A. bisporus/white, Lentinus edodes (Mattila et al. 2002; Teichmann et al. 2007), Craterellus tubaeformis (Teichmann et al. 2007), Tuber melanosporum, T. aestivum and T. indicum (Villares et al. 2012) mushrooms.

Herein, a methodology for sterols extraction and analysis using reversed phase HPLC and ultraviolet detection (HPLC-UV) was optimized and validated. The developed methodology was afterwards applied to quantify ergosterol in wild (Amanita caesarea, Boletus edulis, Cantharellus cibarius, Fistulina hepatica, Lactarius deliciosus, Macrolepiota procera and Morchella esculenta) and cultivated (Agaricus bisporus, Agaricus bisporus Portobello, Flammulina velutipes, Lentinus edodes, Pleurotus eryngii and Pleurotus ostreatus) mushroom species. Species were selected according to their relevance and recognized organoleptic properties. As far as we know, most of these species were analysed regarding their ergosterol content for the first time. 


\section{Materials and methods}

Mushroom species

Seven species of highly appreciated wild mushrooms (Amanita caesarea, Boletus edulis, Cantharellus cibarius, Fistulina hepatica, Lactarius deliciosus, Macrolepiota procera and Morchella esculenta) were collected in Bragança (Northeast Portugal ) in the year 2012 (M. esculenta in April, C. cibarius in May and A. caesarea, B. edulis, F. hepatica, Lactarius deliciosus and Macrolepiota procera in October), and six cultivated mushroom species (Agaricus bisporus, Agaricus bisporus Portobello, Flammulina velutipes, Lentinus edodes, Pleurotus eryngii and Pleurotus ostreatus) were obtained in local grocery stores, also in 2012. The sporocarps were taxonomically identified and representative voucher specimens were placed at the herbarium of Escola Superior Agrária of Instituto Politécnico de Bragança. All the samples were lyophilised (FreeZone 4.5 model 7750031, Labconco, Kansas, USA), reduced to a fine dried powder (20 mesh) and mixed to obtain a homogenised sample. Fat content was determined by Soxhlet extraction (12 h) with hexane.

Standards and reagents

The standards of sterols (ergosterol, cholecalciferol, hexestrol, dietilstilbestrol, 17 $\alpha$ ethynylestradiol, ergocalciferol, dienestrol and cholecalciferol) were purchased from Sigma (St. Louis, MO, USA). Water was treated in a Milli-Q water purification system (TGI Pure Water Systems, USA). Methanol and acetonitrile were of HPLC grade, from 
Lab-Scan (Dublin, Ireland). All other chemicals and solvents were of analytical grade and purchased from common sources.

Sterols extraction and saponification

Method A. Mushroom lipidic fraction was extracted with $n$-hexane refluxing during $2 \mathrm{~h}$ in a Soxhlet apparatus. Each sample was extracted twice ( $\sim 2 \mathrm{~g}$ of a 20 mesh dried powder in each extraction). Before Soxhlet extraction an adequate volume of cholecalciferol (internal standard) was added to each sample. The saponification was performed according to minor modifications of the method applied by Ortíz et al. (Ortíz et al. 2006). Approximately $0.05 \mathrm{~g}$ of the oil sample was transferred to a dark bottle. Ascorbic acid solution $0.1 \mathrm{M}(1 \mathrm{~mL})$ and potassium hydroxide solution $2 \mathrm{M}(5 \mathrm{~mL})$ were added. The samples were saponified shaking the mixture at $125 \mathrm{rpm}$ in a thermostated $\left(60^{\circ} \mathrm{C}\right)$ bath for $45 \mathrm{~min}$. After cooling at room temperature the resulting mixture was filtered and treated with $2.5 \mathrm{~mL}$ of saturated sodium chloride solution and $5 \mathrm{~mL}$ of $n$-hexane. Samples were then stirred for $1 \mathrm{~min}$ in the Vortex mixer. The $n$ hexane phase containing sterols was collected. The aqueous layer was then re-extracted with a new aliquot of $5 \mathrm{~mL} n$-hexane. Both $n$-hexane fractions were combined and dried by passing through anhydrous sodium sulphate. The entire $n$-hexane solution was evaporated (Buchi rotavapor R-210) to dryness in a rotavapor at $40{ }^{\circ} \mathrm{C}$ (Buchi heating bath B-491) and reduced pressure (Buchi vacuum pump V-700 and vacuum controller $\mathrm{V}-850$ ). The resulting residue was dissolved in $1 \mathrm{~mL}$ of methanol and filtered through a $0.45 \mu \mathrm{m}$ nylon syringe membrane. The resultant solution was divided in aliquots and promptly injected into the HPLC system. 
Method B. The applied methodology followed a previously established procedure (Yuan et al. 2007) with minor changes. Dried mushroom powder $(\sim 2 \mathrm{~g})$ was extracted with methanol/dichloromethane $(75: 25, v / v)$ at room temperature in a 1:25 solid to liquid ratio. The extracts were centrifuged at $10,000 \mathrm{rpm}$ for $5 \mathrm{~min}$. The extraction procedure was repeated three times. The total extracts were evaporated to dryness in a rotavapor at $40{ }^{\circ} \mathrm{C}$. The resulting residue was dissolved in $1 \mathrm{~mL}$ of methanol and filtered through a $0.45 \mu \mathrm{m}$ nylon syringe membrane. The resultant solution was divided in aliquots and promptly injected into the HPLC system.

Method $C$. The assayed methodology was performed following the procedure described by Villares et al. (Villares et al. 2012) with slight alterations. Dried mushroom powder $(\sim 2 \mathrm{~g})$ was extracted with $30 \mathrm{~mL}$ of chloroform/methanol mixture $(20: 10, v / v)$ by stirring in an ultrasonic bath at $4{ }^{\circ} \mathrm{C}$ for $30 \mathrm{~min}$. Then, the mixture was centrifuged at $3000 \mathrm{rpm}$ for $10 \mathrm{~min}$. The residue was re-extracted twice and the extracts were combined. The extract was then passed through an Oasis MAX column (Quaternary amine anion sorbent, $150 \mathrm{mg}$, Waters Corp., Milford, MA) preconditioned with $n$ hexane $(8 \mathrm{~mL})$. Sterols and steryl esters were separated from other lipids by passing chloroform $(2 \times 3 \mathrm{~mL})$. Further fractions containing phospholipids and other lipids were discarded. The sample was evaporated to dryness under a nitrogen stream and redissolved in $2 \mathrm{~mL}$ of chloroform. Prior to HPLC analysis samples were filtered through nylon syringe membrane $(0.45 \mu \mathrm{m})$.

Chromatographic analysis 
The analyses were performed by high performance liquid chromatography coupled to ultraviolet detection (HPLC-UV). The HPLC equipment consisted of an integrated system (Knauer, Germany) with a Smartline pump 1000, a degasser system Smartline manager 5000, a Smartline 2500 UV detector and an AS-2057 Plus auto-sampler (Jasco, Japan). Chromatographic separation was achieved with a BGB Analytic AG Inertsil 100A ODS-3 reverse phase column $(4.6 \times 150 \mathrm{~mm}, 5 \mathrm{~mm}$, Knauer $)$ operating at $35{ }^{\circ} \mathrm{C}$ (7971R Grace oven). The mobile phase was acetonitrile:methanol (70:30, v:v), at a flow rate of $1 \mathrm{~mL} / \mathrm{min}$, and the injection volume was $20 \mu \mathrm{L}$; the detection was performed at $280 \mathrm{~nm}$. Ergosterol was quantified by comparison of the area of its peak with the calibration curve obtained from a commercial standard. Cholecalciferol was used as internal standard. The results were expressed in $\mathrm{mg}$ per $\mathrm{g}$ of fat, $\mathrm{mg}$ per $100 \mathrm{~g}$ of dry weight and $\mathrm{mg}$ per $100 \mathrm{~g}$ of fresh weight.

Method validation

Linearity and sensitivity of the HPLC analysis were determined and the method was validated by the instrumental repeatability, precision and accuracy, using $F$. hepatica. This species was selected due to its content in ergosterol, which correspond to the median value among the assayed mushrooms. For linearity, the standard stock solutions were made with hexane from the stock ergosterol solution at seven concentration levels ranging from 31.25 to $2000 \mu \mathrm{g} / \mathrm{mL}$. The calibration curve was obtained by plotting the peak area $v s$. concentration.

The repeatability (intra-day) was checked by analysing $F$. hepatica extract seven consecutive times. For precision assessment, three different extractions of the same sample were injected in triplicate. Precision was calculated as a relative standard 
deviation for the repeated measurements. The accuracy of the method was evaluated by the standard addition procedure (percentage of recovery), with three addition levels $(25$, 50 and $100 \%$ of the peak/area concentration) each one in triplicate. LOD was calculated as the concentration corresponding to 3.3 times the standard error of the calibration curve divided by the slope; LOQ was calculated using the concentration corresponding to ten times the standard error of the calibration curve divided by the slope. The linear regression analysis was carried out by plotting the peak areas against the concentrations of ergosterol.

Statistical analysis

Sterols extraction was performed in triplicate and each sample was injected two times in HPLC-UV. The results are expressed as mean values \pm standard deviation (SD). The differences in ergosterol contents among mushroom species were analysed using oneway analysis of variance followed by Tukey's HSD.

Hierarchical cluster analysis (HCA) was used as an unsupervised learning method to standardized data, checking for similarities between the assayed mushrooms. This method calculates the distances (or correlation) between all samples using a defined metric such as squared Euclidean distance or Chebychev distance. Hierarchical clustering is the most common approach in which clusters are formed sequentially. The most similar objects are first grouped, and these initial groups are merged according to their similarities. Eventually as the similarity decreases all subgroups are fused into a single cluster.

The statistical tests were performed at a 5\% significance level using SPSS v. 18.0 programme. 


\section{Results and discussion}

The majority of methods used to extract lipids might also be effective to extract the sterol fraction. Nonpolar solvents such as $n$-hexane, quantitatively extract free phytosterols and phytosteryl fatty-acid esters. In this work, methods assigned as A and B allowed high purity final extracts as confirmed by the respective chromatograms. Method $\mathrm{C}$ was less effective because the obtained extract retained a higher number of interfering compounds. Among methods A and B, the first was selected due to its higher overall yield, best recovery and repeatability as well as the simple pretreatment. The absorbance was recorded at $280 \mathrm{~nm}$ in the UV detector.

Besides thin layer chromatography techniques, HPLC methods have also been developed to analyze phytosterols, but studies with mycosterols are rather scarce. The analysis of sterols might be performed with polar (e.g. silica, DIOL, amino, CN) or reversed phase (e.g. $\mathrm{C}_{18}=\mathrm{ODS}, \mathrm{C}_{8}$, or phenyl) columns (Moreau et al. 2002). The HPLC methodology herein described was developed with an ODS column, which allowed good peak resolution (Figure 1). Among different assayed proportions of acetonitrile:methanol, the combination 70:30 gave the best results considering elution time and peak resolution. In view of the results there was no need of applying gradient elution, once that the isocratic method allowed good peak resolution. After studying the linearity $(11$ levels $)$ for ergosterol $\left(t_{\mathrm{R}}=13.0 \pm 0.1 \mathrm{~min}\right.$; coefficient of variation, $\mathrm{CV}=$ $0.65 \%)$, a seven-level $(31.25 \mu \mathrm{g} / \mathrm{mL}$ to $2000 \mu \mathrm{g} / \mathrm{mL})$ calibration curve $(\mathrm{y}=0.6566 x+$ $\left.0.01098 ; R^{2}=0.9996\right)$ was made using the peak/area ratio versus concentration of the standard (in $\mu \mathrm{g} / \mathrm{mL}$ ). The average of triplicate determinations for each level was used. 
The validation method was performed using ergosterol, the main sterol in mushrooms. LOD, calculated as the concentration corresponding to 3.3 times the standard error of the calibration curve divided by the slope was $0.3498 \mu \mathrm{g} / \mathrm{mL}$; LOQ, calculated using the concentration corresponding to ten times the standard error of the calibration curve divided by the slope, was $1.060 \mu \mathrm{g} / \mathrm{mL}$. The precision of the equipment was evaluated injecting seven consecutive times the sterols extract of $F$. hepatica. The optimized method proved to be precise $(\mathrm{CV}=1.25 \%)$. Repeatability was assessed by applying the extraction procedure three times to $F$. hepatica powder. The method proved also to be repeatable $(\mathrm{CV}=1.88 \%)$. The method accuracy was evaluated by the standard addition procedure (percentage of recovery). The standard mixture was added to the samples in three concentration levels $(25,50$ and $100 \%$, i.e. 250,500 and $1000 \mu \mathrm{g} / \mathrm{mL}$, of the peak/area concentration, each one in triplicate) before the extraction. The method showed good recovery for the three added levels: $25 \%-96 \pm 1 \% ; 50 \%-92 \pm 2 \% ; 100 \%$ $92 \pm 2 \%$ ). All chromatographic determinations were performed following the principles defined in $\mathrm{AOAC}$, falling in the range defined by AOAC peer verified methods program (1998).

The results obtained for fat contents were quite similar with previously published results, either in cultivated mushrooms (Reis et al. 2012) as well as in wild species: $A$. caesarea (Reis et al. 2011), B. edulis (Heleno et al. 2011), C. cibarius (Barros et al. 2008), F. hepatica (Heleno et al. 2009), L. deliciosus (Barros et al. 2007) and $M$. procera (Barros et al. 2007).

Figure 1 shows the sterols profile of $F$. hepatica. Ergosterol is clearly the most abundant steroid, but its content varied greatly among species (Table 1), as it was previously indicated using similar (chanterelle, crimini, enoki, maitake, morel, oyster, portobello, vitamin D-enhanced Portobello, shiitake and white button) mushrooms 
(Phillips et al. 2011). For comparative purposes, ergosterol concentrations were expressed in three different units (mg per $\mathrm{g}$ of fat, $\mathrm{mg}$ per $\mathrm{g}$ of dried mushroom and $\mathrm{mg}$ per $g$ of fresh mushroom). The obtained values are in the same range as the results published elsewhere (Mattila et al. 2002; Villares et al. 2012), despite being quite lower than the values obtained by Teichmann et al. (Teichmann et al. 2007). A. bisporus showed the highest ergosterol content when expressed in $\mathrm{mg} / \mathrm{g}$ of fat $(158 \pm 2)$ or in $\mathrm{mg} / 100 \mathrm{~g}$ of dry weight $(352 \pm 1)$. Nevertheless, the maximum value in $\mathrm{mg} / 100 \mathrm{~g}$ of fresh weight was found in L. edodes (44.0 \pm 0.3$)$. Otherwise, M. esculenta presented the lowest content independently of the units: $9.9 \pm 0.3 \mathrm{mg} / \mathrm{g}$ of fat; $43 \pm 2 \mathrm{mg} / 100 \mathrm{~g}$ of dry weight; $4.0 \pm 0.3 \mathrm{mg} / 100 \mathrm{~g}$ of fresh weight. These differences in mushrooms assortment result from the different values of fat and water percentages for each species. Since cultivated mushrooms tended to have higher ergosterol contents, a HCA was applied to verify some particular disimilarity among wild and cultivated mushrooms. The results obtained are shown as a dendrogram (Figure 2) in which is easily seen that both types of mushrooms were not clustered separately. In this analysis, samples are grouped in clusters in terms of their nearness or similarity, defined in this case by the amounts or ergosterol in each mushroom species. The mixture of cultivated and wild mushroom in the two primarily formed clusters indicates that the differences in ergosterol contents are not strong enough for their separation. The ergosterol content in A. bisporus Portobello, for instance, placed this mushroom together with the wild species; this result can be considered as an evidence of the influence exerted by mushroom maturation over the ergosterol content (Villares et al., 2012).

The knowledge acquired in ergosterol quantification might represent an important step to understand the real effects of consuming foods naturally containing or enriched with this sterol. In fact, the health-promoting properties of ergosterol, including the 
antioxidant (Shao et al. 2010), anti-inflammatory (Kuo et al. 2011) antihyperlipidemic (Hu et al. 2006), genetic expression control (Lochman and Mikes 2005) and antitumoral activities (Takaku et al. 2001; Yazawa et al. 2000), are closely related to the uptake doses. Furthermore, it will be important to understand how its concentration varies during the main fungi development stages.

Overall, a reproducible and accurate HPLC-UV technique was fully developed. Besides ergosterol quantification, the resulting signals presented good resolution, indicating the suitability of this methodology to assess sterol profiles in future studies. Furthermore, this methodology is faster than the widely used GLC analysis and operates under milder column temperatures and non-destructive detection conditions. Moreover, the chosen extraction and saponification steps do not require complex conditions, being suitable for routine analysis. Hence, the employed method proved to be a useful tool for rapidly determining sterol profile, compared with other chromatographic methodologies and sample preparation protocols. HPLC analysis was performed with little sample purification, preventing unwanted samples losses. Regarding ergosterol contents in the assayed species, despite a complete separation could not be obtained among cultivated and wild mushrooms, ergosterol tended to be higher in cultivated species, with relevance for A. bisporus, the most consumed mushroom worldwide.

\section{Acknowledgements}

The authors are grateful to Fundação para a Ciência e a Tecnologia (FCT, Portugal) and

COMPETE/QREN/EU for the financial support of this work (research project PTDC/AGRALI/110062/2009) and to CIMO (strategic project PEst- 
OE/AGR/UI0690/2011). J.C.M. Barreira also thanks to FCT, POPH-QREN and FSE for his grant (SFRH/BPD/72802/2010.

\section{Compliance with Ethics Requirements}

\section{Conflict of Interest}

João C.M. Barreira declares that he has no conflict of interest.

M. Beatriz P.P. Oliveira declares that she has no conflict of interest.

Isabel C.F.R. Ferreira declares that she has no conflict of interest.

João C.M. Barreira has a research grant from FCT.

None of the authors have a financial relationship with the organization that sponsored the research.

This article does not contain any studies with human or animal subjects.

\section{References}

AOAC, Peer-verified methods program, Manual on policies and procedures (1998) AOAC International, Arlington, VA, USA.

Bada JC, Camacho ML, Prieto M, Alonso L (2004). Characterization of oils of hazelnuts from Asturias, Spain. Eur J Lipid Sci Tech 106:294-300 
Barros L, Baptista P, Correia DM, Casal S, Morais JS, Ferreira ICFR (2007) Effects of conservation treatment and cooking on the chemical composition and antioxidant activity of Portuguese wild edible mushrooms. J Agr Food Chem 55:4781-4788

Barros L, Baptista P, Correia DM, Casal S, Oliveira MBPP, Ferreira ICFR (2007) Fatty acid and sugar compositions, and nutritional value of five wild edible mushrooms from Northeast Portugal. Food Chem 105:140-145

Barros L, Venturini BA, Baptista P, Estevinho LM, Ferreira ICFR (2008) Chemical composition and biological properties of portuguese wild mushrooms: a comprehensive study. J Agr Food Chem 56:3856-3862

Giacometti J (2001) Determination of aliphatic alcohols, squalene, $\alpha$-tocopherol and sterols in olive oils: direct method involving gas chromatography of the unsaponificable fraction following silylation. Analyst 126:472-475

Heleno SA, Barros L, Sousa MJ, Martins A, Ferreira ICFR (2009) Study and characterization of selected nutrients in wild mushrooms from Portugal by gas chromatography and high performance liquid chromatography. Microchem J 93: 195-199

Heleno SA, Barros L, Sousa MJ, Martins A, Santos-Buelga C, Ferreira ICFR (2011) Targeted metabolites analysis in wild Boletus species. LWT-Food Sci Technol $44: 1343-1348$

Hu SH, Liang ZC, Chia YC, et al. (2006) Antihyperlipidemic and antioxidant effects of extracts from Pleurotus citrinopileatus. J Agr Food Chem 54:2103-2110.

Kritchevsky D, Shirley CC (2005) Phytosterols - health benefits and potential concerns: a review. Nutr Res 25:413-428 
Kuo CF, Hsieh CH, Lin WY (2011) Proteomic response of LAP-activated RAW 264.7 macrophages to the anti-inflammatory property of fungal ergosterol. Food Chem $126: 207-212$

Lagarda MJ, García-Llatas G, Farré R (2006) Analysis of phytosterols in foods. J Pharmaceut Biomed 41:1486-1496

Lochman J, Mikes V (2005) Activation of different defence-related genes expression by ergosterol. FEBS J 272:470

Manzi P, Panfili G, Pizzoferrato L (1996) Normal and reversed phase HPLC for more complete evaluation of tocopherol, retinols, carotenes and sterols in dairy products. Chromatographia 43:89-93

Mattila P, Lampi A-M, Ronkainen R, Toivo J, Piironen, V (2002) Sterol and vitamin $\mathrm{D}_{2}$ contents in some wild and cultivated mushrooms. Food Chem 76:293-298

Moreau RA, Whitaker BD, Hicks KB (2002) Phytosterols, phytostanols, and their conjugates in foods: structural diversity, quantitative analysis, and healthpromoting uses. Prog Lipid Res 41:457-500

Ortíz CML, Moya SMP, Navarro VB (2006) A rapid chromatographic method for simultaneous determination of $\beta$-sitosterol and tocopherol homologues in vegetable oils. J Food Compos Anal 19:141-149

Parks LW, Weete JD (1991) Fungal sterols. In GW Patterson and WD Nes (Eds.), Physiology and biochemistry of sterols. Champaign, IL: American Oil Chemists' Society

Phillips KM, Ruggio DM, Horst RL, et al. (2011) Vitamin D and sterol composition of 10 types of mushrooms from retail suppliers in the United States. J Agr Food Chem 59:7841-7853 
Reis FS, Barros L, Martins A, Ferreira ICFR (2012) Chemical composition and nutritional value of the most widely appreciated cultivated mushrooms: An interspecies comparative study. Food Chem Toxicol 50:191-197

Reis FS, Heleno SA, Barros L, et al. (2011) Towards the antioxidant and chemical characterization of mycorrhizal mushrooms from northeast Portugal. J Food Sci 76: $824-830$

Senatore F (1992) Chemical constituents of some mushrooms. J Sci Food Agr 58:499503

Shao SQ, Hernandez M, Kramer JKG, Rinker DL, Tsao R (2010) Ergosterol profiles, fatty acid composition, and antioxidant activities of button mushrooms as affected by tissue part and developmental stage. J Agr Food Chem 58:11616-11625

Takaku T, Kimura Y, Okuda H (2001) Isolation of an antitumor compound from Agaricus blazei Murill and its mechanism of action. J Nutr 131:1409-1413

Teichmann A, Dutta PC, Staffas A, Jägerstad M (2007) Sterol and vitamin $\mathrm{D}_{2}$ concentrations in cultivated and wild grown mushrooms: Effects of UV irradiation. LWT-Food Sci Technol 40:815-822

Villares A, García-Lafuente A, Guillamón E, Ramos Á (2012) Identification and quantification of ergosterol and phenolic compounds occurring in Tuber spp. truffles. J Food Compos Anal 26:177-182

Yazawa Y, Yokota M, Sugiyama K (2000) Antitumor promoting effect of an active component of Polyporus, ergosterol and related compounds on rat urinary bladder carcinogenesis in a short-term test with concanavalin A. Biol Pharm Bull 23:1298-1302 
Yuan J-P, Wang J-H, Liu X, Kuang, H-C, Zhao S-Y (2007) Simultaneous determination of free ergosterol and ergosteryl esters in Cordyceps sinensis by HPLC. Food Chem 105:1755-1759 
Table 1. Fat percentage and ergosterol content in cultivated and wild edible mushrooms. Results are showed as mean \pm SD $(n=3)$. In each column, different letters mean significant differences.

Figure 1. HPLC-UV sterols profile of Agaricus bisporus. 1- internal standard (cholecalciferol) added at $200 \mu \mathrm{g} / \mathrm{mL}$; 2- ergosterol.

Figure 2. Dendrogram obtained with Z-scores standardization results after applying the Ward linkage method. (C)- cultivated mushrooms; (W)- wild mushrooms. 
Table 1.

\begin{tabular}{|c|c|c|c|c|}
\hline Species & Fat $(\%)$ & Ergosterol (mg/g fat) & Ergosterol $(\mathrm{mg} / 100 \mathrm{~g} \mathrm{dw})$ & Ergosterol $(\mathrm{mg} / 100 \mathrm{~g} \mathrm{fw}$ \\
\hline Agaricus bisporus & $2.22 \pm 0.03 \mathrm{c}$ & $158 \pm 2 \mathrm{a}$ & $352 \pm 1 \mathrm{a}$ & $30.8 \pm 0.1 \mathrm{~b}$ \\
\hline Agaricus bisporus Portobello & $1.15 \pm 0.04 \mathrm{j}$ & $67 \pm 2 \mathrm{~g}$ & $77 \pm 1 \mathrm{~h}$ & $6.43 \pm 0.1 \mathrm{j}$ \\
\hline Flammulina velutipes & $1.94 \pm 0.02 \mathrm{de}$ & $98 \pm 2 \mathrm{~d}$ & $189 \pm 2 \mathrm{~d}$ & $22.9 \pm 0.2 \mathrm{~d}$ \\
\hline Lentinus edodes & $1.99 \pm 0.04 \mathrm{~d}$ & $109 \pm 3 \mathrm{c}$ & $217 \pm 2 \mathrm{c}$ & $44.0 \pm 0.3 \mathrm{a}$ \\
\hline Pleurotus eryngii & $1.85 \pm 0.03 \mathrm{ef}$ & $101 \pm 1 \mathrm{~d}$ & $187 \pm 1 \mathrm{~d}$ & $20.5 \pm 0.1 \mathrm{e}$ \\
\hline Pleurotus ostreatus & $1.43 \pm 0.02 \mathrm{~h}$ & $73 \pm 2 \mathrm{fg}$ & $104 \pm 1 \mathrm{~g}$ & $11.3 \pm 0.1 \mathrm{~h}$ \\
\hline Amanita caesarea & $3.53 \pm 0.05 \mathrm{~b}$ & $66 \pm 1 \mathrm{~g}$ & $231 \pm 1 \mathrm{~b}$ & $21.1 \pm 0.1 \mathrm{e}$ \\
\hline Boletus edulis & $1.75 \pm 0.03 \mathrm{f}$ & $134 \pm 2 b$ & $234 \pm 2 b$ & $25.4 \pm 0.2 \mathrm{c}$ \\
\hline Cantharellus cibarius & $1.23 \pm 0.03 \mathrm{ij}$ & $105 \pm 4 \mathrm{~cd}$ & $129 \pm 1 \mathrm{e}$ & $9.9 \pm 0.1 \mathrm{i}$ \\
\hline Fistulina hepatica & $1.32 \pm 0.03 \mathrm{hi}$ & $82 \pm 1$ e & $108 \pm 1 \mathrm{~g}$ & $13.5 \pm 0.1 \mathrm{f}$ \\
\hline Lactarius deliciosus & $1.95 \pm 0.04 \mathrm{de}$ & $28 \pm 1 \mathrm{~h}$ & $55 \pm 1 \mathrm{i}$ & $5.5 \pm 0.1 \mathrm{k}$ \\
\hline Macrolepiota procera & $1.58 \pm 0.03 \mathrm{~g}$ & $75 \pm 1$ ef & $118 \pm 2 \mathrm{f}$ & $12.0 \pm 0.2 \mathrm{~g}$ \\
\hline Morchella esculenta & $4.34 \pm 0.05 \mathrm{a}$ & $9.9 \pm 0.3 \mathrm{i}$ & $43 \pm 2 j$ & $4.0 \pm 0.21$ \\
\hline
\end{tabular}




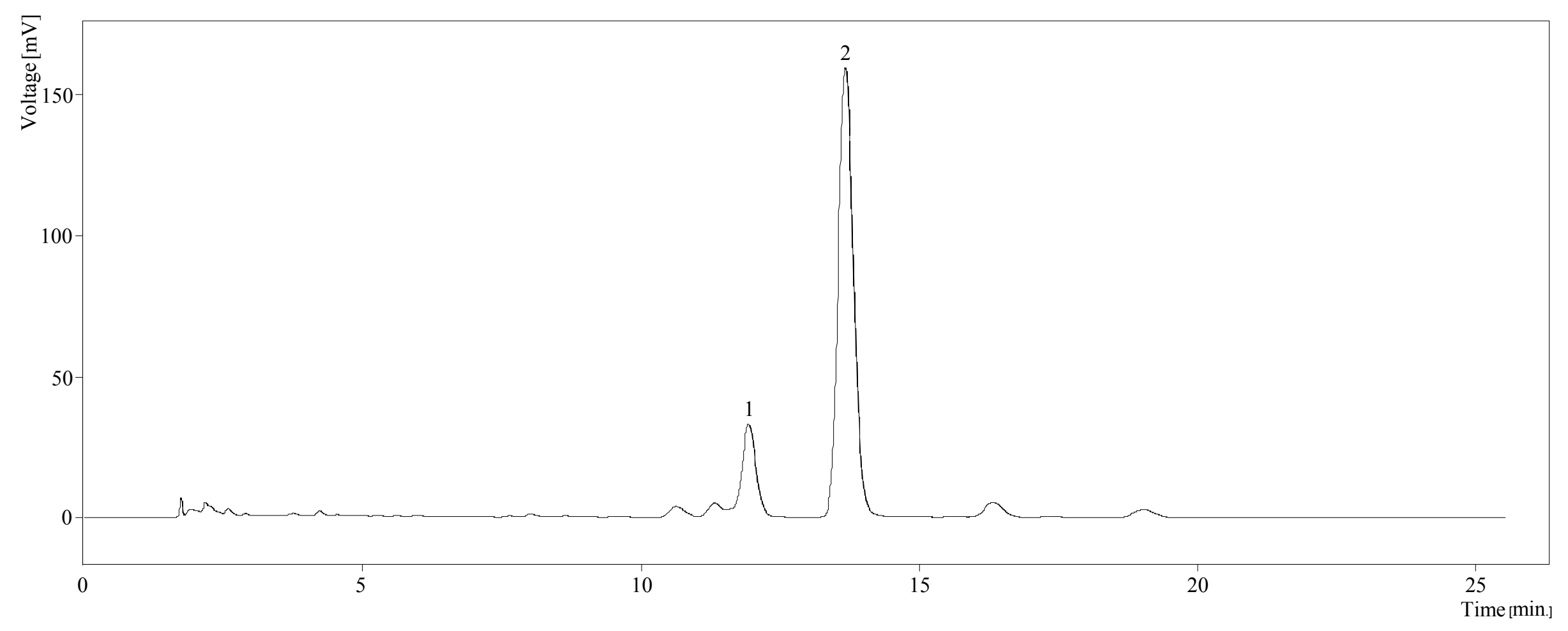

Figure 1. 


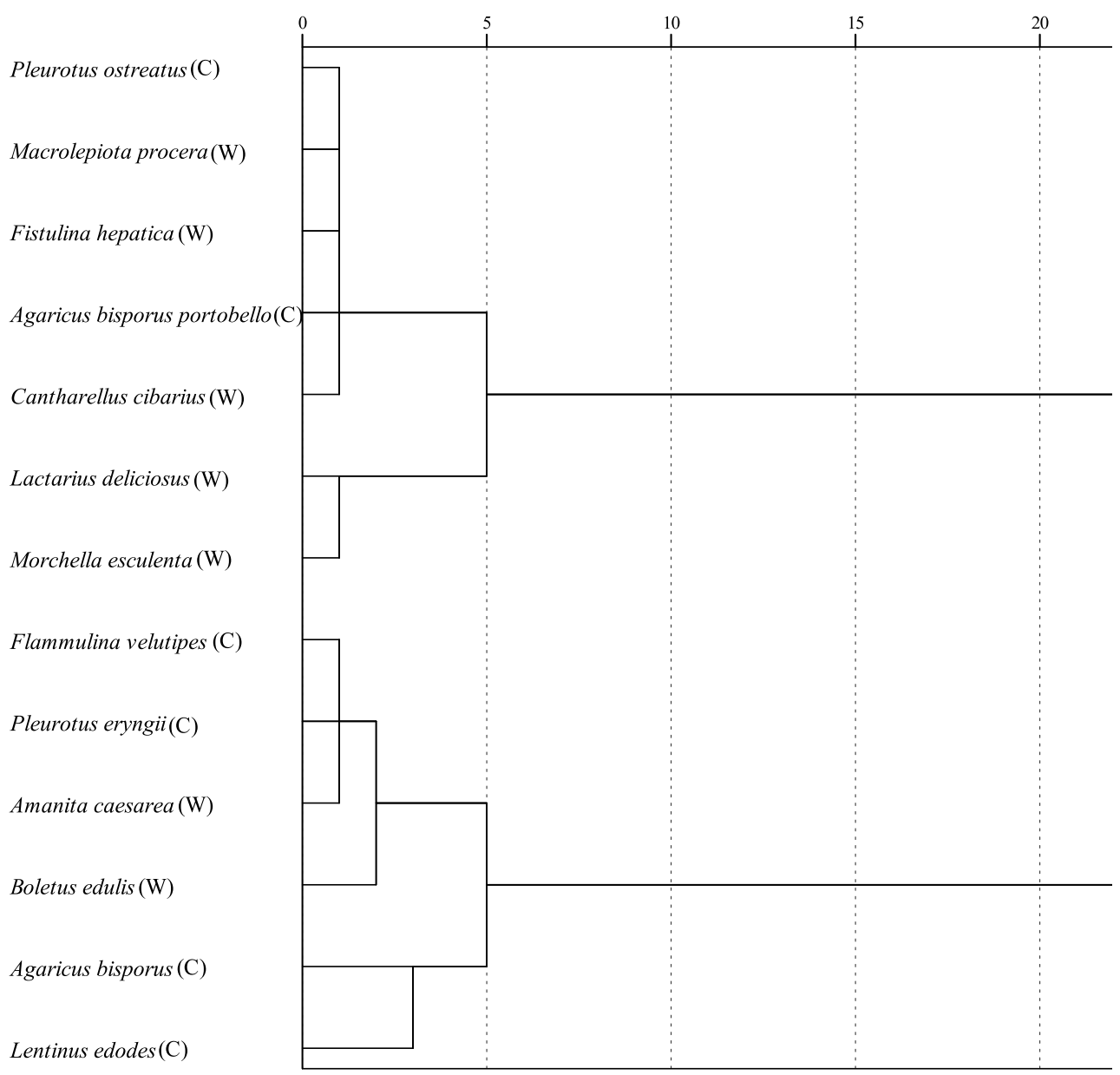

Figure 2. 